\title{
Duration of Hospital Admission, Need of On-demand Analgesia and other Peri- procedural and Short-Term Outcomes in Sub-Cutaneous vs. Transvenous Implantable Cardioverter Defibrillators.
}

Serge Boveda ${ }^{1}$, MD., Tej Elbanet Chalbia ${ }^{1}$, MD., Sophie Jacob², PhD., Stéphane Combes ${ }^{1}$, MD., Nicolas Combes ${ }^{1}$, MD., Christelle Cardinㅜ, MD., Guillaume Laborie ${ }^{1}$, MD., Maria Joao Sousa ${ }^{1}$, MD., Zeynab Jebberi ${ }^{1}$, MD., Sophia Mzoughi ${ }^{1}$, MD., Jean-Paul Albenque ${ }^{1}$, MD., Rui Providencia ${ }^{3}$, MD., $\mathrm{PhD}$.

1 Département de Rythmologie, Clinique Pasteur, 45 avenue de Lombez, 31300 Toulouse, France. All these authors take responsibility for all aspects of the reliability and freedom from bias of the data presented and their discussed interpretation

2 IRSN, Laboratory of Epidemiology, PSE-SANTE, SESANE, LEPID, BP17, 92262 Fontenay-auxRoses, France. This author takes responsibility for all aspects of the reliability and freedom from bias of the data presented and their discussed interpretation.

${ }^{3}$ St. Bartholomew's Hospital, Barts Heart Centre, West Smithfield, London, EC1A 7BE, UK. This author takes responsibility for all aspects of the reliability and freedom from bias of the data presented and their discussed interpretation.

\section{Corresponding author:}

Serge Boveda, MD, Cardiologie, Département de Rythmologie, Clinique Pasteur 45 avenue de Lombez, 31300 Toulouse, France

Phone: 335622116 45; Fax: 335622116 41;

Email: sboveda@clinique-pasteur.com

Conflicts of Interest: S. Boveda is consultant for Medtronic, Boston Scientific, and Livanova. JP. Albenque is consultant for Abbott and Biosense Webster. 
Keywords: Subcutaneous Implantable Defibrillator; Transvenous ICD; pain assessment; duration of hospitalization; short term clinical outcomes; matched comparison

\section{Abstract:}

Background: Post-procedural recovery following Sub-cutaneous ICD (S-ICD) implantation is feared to be more painful and to require more prolonged hospital admission. The purpose of this study was to compare peri-procedural and short clinical outcomes of the S-ICD vs. the Transvenous ICD (TV-ICD).

Methods: We conducted a single-center cross-sectional study including all consecutive patients who underwent S-ICD implantation by the same operator since January 2016 and a gender and age-matched control group with all single chamber TV-ICD implanted patients over a contemporary time period.

Results: Thirty-one patients (sex ratio $1 / 5$; mean age $58.7 \pm 13.2$ years) with S-ICD were compared to 31 matched TV-ICD patients. Duration of the implant procedure was significantly longer for the S-ICD $(58.0 \pm 24.4 \mathrm{~min}$ vs $41.7 \pm 20.8 \mathrm{~min}$ TV-ICD, $\mathrm{P}<0.01)$. Mean fluoroscopy time for the TV-ICD was $3.5 \pm 3.6$ minutes vs $0.1 \pm 0.01$ minutes for all S-ICD patients $(p<0.01)$. Requirement of on-demand analgesia administration, and duration of hospitalization (1.5 days for both groups; $p=N S$ ) were similar in the two groups. No peri-procedural events were reported, and after a mean follow-up of 6 months, the only complication was a pocket infection requiring reintervention in the TV-ICD group.

Conclusions: The S-ICD appears to be as effective and safe as the conventional single chamber TV-ICD. Duration of hospital admission and need of on-demand analgesia are also comparable for S-ICD patients. 


\section{Introduction}

The Subcutaneous Implantable Defibrillator (S-ICD) is a promising technology developed to overcome many of the limitations of the conventional Transvenous single-chamber ICD's (TV-ICD) [1, 2].

Based on several randomized clinical studies that demonstrated a low complication rate and an excellent efficacy for conversion of ventricular tachyarrhythmias [3], a Class IIa recommendation for the S-ICD has been added to the most recent European Society of Cardiology (ESC) Guidelines as an alternative to the TV-ICD, in the absence of pacing or resynchronization indication [4].

However, and despite the fact that most ICDs do not have a pacing or CRT indication, the TV-ICD is still the most widely used in most of the European centers [5]. Interestingly, the rationale for preferring a TV-ICD is generally not supported by strong reasons, such as the need for permanent pacing or anti-tachycardia pacing, and is not always very clear. This behavior may be explained by the lack of experience or availability of the S-ICD in some centers, but also by a more conservative approach by physicians who are still not entirely convinced of the real-world results of this technology, or may think that this procedure is associated with a more painful and prolonged recovery, as a result of the extensive sub-cutaneous tunnelization for the ICD lead and the different pocket location.

In this study, we aimed to compare peri-procedural and clinical outcomes after implantation of the S-ICD or the TV-ICD based on a cross-sectional study including SICD implanted patients and an age and gender-matched control group of patients implanted with TV-ICD, with focus on procedure duration, pain assessment and duration of hospitalization, perioperative complications and short-term follow-up. 


\section{Methods}

We conducted a cross-sectional study, including all consecutive patients who underwent S-ICD implantation, from January to December 2016 at Clinique Pasteur, Toulouse, France, and an age and gender-matched control group of patients implanted with TVICD system in the same center. The study complies with the ethical guidelines of the 1975 Declaration of Helsinki. All patients gave written informed consent allowing the use of their medical data.

\subsection{Patient Selection}

Patients implanted with an S-ICD were compared with a similar number of controls implanted during the same time period, with a conventional TV-ICD system. The "nearest neighbor" method was used for matching S-ICD cases and TV-ICD controls, and for each S-ICD patient, we assigned the first subsequent gender and age-matched, single-chamber TV-ICD patient implanted within a time-interval of 30 days, without pacing or CRT indication. All the devices included in this study were implanted by the same operator, having an experience of more than 20 years for the TV-ICD and more than 2 years for the S-ICD.

All patients had an indication for ICD implantation according to the American College of Cardiology/American Heart Association [6] and European Society of Cardiology guidelines [4] for primary and secondary prevention of cardiac arrhythmias (see Figure 1).

Screening was performed in all S-ICD candidates $[1,2]$. This consisted of using a dedicated screening tool to confirm their eligibility by analyzing the surface electrocardiogram signals in both supine and standing positions. In patients passing screening, a detailed discussion with the implanting physician about the pros and cons 
of both technologies took place, selection of the device was performed in accordance with the patient's preference. Written informed consent was obtained for all patients.

Importantly, the only exclusion criteria for this S-ICD vs. TV-ICD comparison was requirement of pacing and/or resynchronization requirement.

The Emblem ${ }^{\mathrm{TM}}$ S-ICD (Boston Scientific $@$, St. Paul, MN) was implanted in all cases. In the TV-ICD group, all devices were single-chamber, and all existing manufacturers and models were used.

\subsection{Procedure}

Because of Departmental regulation in our Institution, general anesthesia was used in all TV-ICD and S-ICD implantation procedures. General anesthesia management was at the discretion of the anesthetists. However, deep sedation with free ventilation was preferred when possible. The TV-ICDs were implanted in a pre-pectoral pocket. The SICDs were implanted in an intermuscular pocket, between the large serratus and the dorsal muscles. For the S-ICD, the left parasternal position of the shock coil with the pulse generator positioned over the sixth rib, in the left mid-axillary line, was recognized as the optimal configuration, and the incision strategy included a two incisions (left latero-thoracic and sub-xyphoidal) technique in all patients.

All S-ICD patients underwent a standardized intraoperative defibrillation test. The first shock energy was programmed to $65 \mathrm{~J}$, resulting in a safety margin of at least $15 \mathrm{~J}$. In case of ineffective first shock delivery, the second shock energy was set to $80 \mathrm{~J}$ with reversed polarity. In the event of failed cardioversion/defibrillation after the second internal shock, external defibrillation was attempted. As it is now a consensus not performing Defibrillation Threshold Testing (DFT) shock systematically [7], we only performed DFT, in the TV-ICD group, to specific patients for secondary prevention. 
All patients had their devices interrogated the day after implantation, and optimization of detection vectors in 2 positions (sitting and lying) was performed in S-ICD patients. A chest X-ray was obtained the first postoperative day after implantation to confirm stable lead position in all patients.

After the operation, we used a post-procedure pain management protocol where oral paracetamol (maximum dose: $4000 \mathrm{mg} /$ day), oral tramadol (maximum dose: $400 \mathrm{mg} /$ day), and endovenous or subcutaneous morphine (maximum dose: $0.5 \mathrm{mg} / \mathrm{Kg} / \mathrm{day}$ ) were given on-demand, and in escalation according to the patient's level of pain.

After discharge, an exercise test was performed 4 weeks after implantation, and sensing vectors were checked again for preventing from T-wave oversensing during exertion in all S-ICD patients.

The programming of ventricular arrhythmias therapies was standardized to prevent inappropriate treatments: a shock only zone $\geq 220 \mathrm{bpm}$ was set in both groups. Additionally, a monitor zone between 170 and 219 bpm with prolonged detection time was also programmed in the TV-ICD group.

\subsection{Patient follow-up, clinical outcomes and study endpoints}

Anthropometric and clinical data was collected for all patients, as well as information on procedural and/or peri-procedural complications. Total hospitalization duration in days (patients were always admitted the day before the operation, and kept at least for a night following the procedure for logistical reasons), and procedural (general anesthesia induction included) and fluoroscopy duration in minutes were collected (Figure 1).

Pain management was assessed during the initial 24 hours after implantation through comparison of the required doses of on-demand analgesia as requested by the patient. 
Follow-up information was obtained following an initial outpatient clinical appointment at our center, 4 weeks after implantation for the exercise test (S-ICD patients only), 3 months after discharge (all patients), by remote monitoring, and through regular appointments every 4 to 6 months for device evaluation (all patients).

Different endpoints assessed during follow-up included: pocket/incision healing process, occurrence of appropriate therapies, inappropriate therapies and other complications (i.e. lead dislodgement or failure, etc...), as well as overall and specific mortalities. Device interrogation printouts were checked by the lead-investigator for appropriate and inappropriate ICD therapy.

\subsection{Statistical analysis}

Descriptive and inferential statistics were performed using IBM SPSS Statistics 21.0 for Windows (IBM Corp, Somers, NY). Pairwise comparisons of continuous variables between both groups (S-ICD patients and matched TV-ICD controls) were made using the Student paired t-test. The Fisher's exact test, which is adapted when dealing with small counts in contrast with Chi-square test, was performed to compare the differences in frequencies between groups. $P<.05$ was considered significant for all analyses.

\section{Results}

Thirty-one consecutive patients (sex ratio $1 / 5$; mean age $58.7 \pm 13.2$ years) received an S-ICD and were matched to 31 TV-ICD controls.

Clinical parameters for all enrolled patient are summarized in Table 1. The prevalence of a primary prevention indication was similar in both groups (58\% S-ICD vs. $64 \%$ TV-ICD patients; $p=0.60)$. 
In the S-ICD arm, eight patients had a previous ICD which was abandoned, due to lead dysfunction (5 patients) or extracted, due to infection (3 patients).

Procedure duration was significantly longer for the S-ICD $(58.1 \pm 24.4 \mathrm{~min}$ S-ICD vs 41.7 \pm 20.8 min TV-ICD; $\mathrm{P}<0.01)$. Conversely, fluoroscopy duration was shorter $(0.1 \mathrm{~min} \pm$ 0.01 ) in all S-ICD patients (vs. $3.5 \pm 3.6$ min with the TV-ICD; $P=0.001,>95 \%$ reduction) as the implantation was guided by anatomical landmarks, and screening was only required to confirm the final position of the lead.

No procedural or peri-procedural complications were observed in any of the two groups. However, failure to obtain venous access for the TV-ICD lead was observed in 2 cases, and a contralateral implant was performed during the same session. No cases of pneumothorax, or haemothorax were observed.

Ventricular fibrillation was effectively terminated by the first programmed 65 Joules subcutaneous shock in all S-ICD patients.

Requirement of analgesia, as assessed by on-demand administered doses of paracetamol, morphine and tramadol was comparable in the two groups $(P>0.05)$ (Table 2).

All patients were discharged within a mean of 1.5 days after the implantation procedure $(p>0.05)$ (Table 2). The maximum length of stay was of 4 and 5 days respectively in the S-ICD and the TV-ICD groups. In neither of these cases was due refractory pain the culprit for the prolonged admission: an administrative problem occurred with an S-ICD patient who could not be transferred to his home hospital, and a hematoma requiring treatment with pressure dressing in the TV-ICD group.

After a mean follow-up of 6 months, the only complication was a pocket infection diagnosed 3 weeks after implantation, requiring device extraction and delayed reimplantation in the TV-ICD group. No inappropriate shocks were observed in any 
patients during this period. In addition, two patients implanted with an S-ICD received an appropriate therapy for ventricular arrhythmia during this follow-up.

\section{Discussion}

Our data show comparable short-term performance between the S-ICD and the TV-ICD systems, with regard to efficacy and safety. Even though there were no puncturerelated complications (haemothorax or pneumothorax), venous access was not possible in 2 TV-ICD patients and a contra-lateral device implant was required, leading to an extra infra-clavicular incision. Additionally, the S-ICD was associated with minimal use of fluoroscopy, which can translate in long-term benefit both for the patient and operator $[8,9]$. Finally, our data on prescribed analgesia suggest that the experienced pain during the recovery period immediately following the implant is comparable for both groups, as well as the duration of hospitalization. Both devices can be implanted in less than 1 hour, but TV-ICDs skin-to-skin time was 15 minutes shorter. These findings are of importance, as these three factors (lower radiation exposure, no need of venous access, and therefore no puncture-related complications or failure to obtain venous access, and comparable post-procedural recovery), should also be weighted when considering an ICD and need to be an important part of the decision process.

\subsection{Efficacy and Safety}

Many physicians routinely implant TV-ICDs in patients without a pacing indication. The rationale of the S-ICD is to decrease the long-term risk lead failure or infection associated with transvenous defibrillation leads [1]. The question is whether the short- 
term advantages provided by the S-ICD could also be convincing enough in terms of safety and efficacy, in order to change operators' habits.

Some operators are concerned about the lack of anti-tachycardia pacing in the S-ICD. However, in MADIT-RIT, a prospective randomized trial of 1500 patients, designed to determine if alternate anti-tachycardia programming could reduce the occurrence of inappropriate therapy in primary prevention patients with transvenous systems, programming of ICD therapies for rates $\geq 200 \mathrm{bpm}$ as compared with conventional programming at the time, was associated with a reduction in inappropriate therapies and all-cause mortality during long-term follow-up [10].

Pooling the 2 largest prospective studies of the S-ICD, IDE and EFFORTLESS, Burke et al. evaluated a large database with 882 patients who received S-ICDs, followed for $651 \pm 345$ days. The success of shock therapy after up to 5 shocks for ventricular tachy-arrhythmias was $98.2 \%$, and the estimated 3-year inappropriate shock rate was $13.1 \%$ [11]. In addition, the use of the latest detection algorithm is independently associated with a lower rate of inappropriate shock delivery in S-ICD patients [12].

There is currently scarce data available comparing S-ICDs and TV-ICDs. The START study, a head-to-head comparison between the S-ICD and the TV-ICD, showed that appropriate ventricular arrhythmia detection was excellent for all ICD systems; however, specificity of supraventricular arrhythmia discrimination by the S-ICD was superior to discrimination by the TV-ICD (98.0\%[S-ICD] vs 76.7\%[SC-TV range: $64.0-92.0 \%$ ] vs 68.0\%[DC-TV range: 32.7-89.8\%; $P<0.001]$ ] [13].

Honarbakhsh et al. compared 69 S-ICD cases to 69 TV-ICD controls during a mean follow-up of 32 months. A higher rate of total device-related complications was observed in the TV-ICD group ( $n=20,29 \%$ vs. $n=6,9 \% ; p=0.004)$. This occurred predominantly as a result of transvenous lead-related complications [14]. 
A retrospective study from the Netherlands compared long-term clinical outcomes in a cohort of 140 patients with S-ICDs vs. 140 TV-ICDs controls. The complication rate at 5years follow-up in S-ICD and TV-ICD patients was comparable (13\% and $18 \%$ respectively; $p=0.80$ ), but complication type differed. Lead complications occurred more often in the TV-ICD group ( $11.5 \%$ vs. $0.8 \%$ S-ICD patients; $p=0.03)$. S-ICD patients had more non-lead-related complications (pocket erosion, defibrillation threshold testing failure, and device failure) than TV-ICD patients ( $9.9 \%$ vs $2.2 \% ; \mathrm{p}=0.04)$. Rates of appropriate and inappropriate shocks were similar between the 2 groups at 5-year of follow-up. Appropriate shock rates occurred in $17 \%$ of S-ICD patients vs. $21.3 \%$ of TVICD controls $(p=0.36)$. The rate of inappropriate shocks was comparable $(20.5 \%$ S-ICD vs. $19.1 \%$ TV-ICD; $p=0.64$ ). Inappropriate shocks from TV-ICDs were mainly due to supraventricular tachycardia (94\%), while in S-ICD patients these were due to oversensing in $85 \%$ and supraventricular tachycardia in $15 \%$ of cases [15].

In these matched cohorts of S-ICD and TV-ICD patients, where most transvenous systems were dual-chamber, complication rates were almost similar. We have observed similar findings, even if in our cohort, we aimed to compare exclusively the S-ICD with the single chamber TV-ICD, which is the simplest trans-venous device.

\subsection{Procedure and fluoroscopy duration}

In the EFFORTLESS S-ICD Registry the average procedure time ('skin to skin') was 69 \pm 27 min (median $61 \mathrm{~min}$ ), which is comparable to our study [4].

In a study by Köbe et al., mean implantation time was also longer for S-ICDs but no significant difference was observed with trans-venous devices procedures [16], like in our experience. 
Even if some argue that the S-ICD is technically more difficult and time-consuming than the TV-ICD implantation, this is not confirmed by the literature $[3,16]$. Our study results suggest the same, as all implantations were performed by the same operator and a comparable procedural duration was observed among the two interventions. One could also argue that these data still refer to the initial experience with the S-ICD, while the TV-ICD is being routinely implanted for a long time, which can explain the longer procedure duration with the most recent technology. In addition, the systematic defibrillation threshold testing for S-ICD patients may partly explain the trend for a longer procedure duration in this group. Even though the S-ICD takes 15-minutes longer in average to be implanted, both procedures can be done in less than an hour.

On the other hand, there is a clear and very significant difference in terms of fluoroscopy time and radiation exposure when comparing both devices, and this last point is clearly in favour of the S-ICD.

\subsection{Pain Management and Length of stay after S-ICD implantation}

To the best of our knowledge, this study is the first to compare post-operative pain management between the conventional TV-ICD and the S-ICD. Interestingly and maybe surprisingly, despite a larger pocket, two incisions and extensive sub-cutaneous tunnelization for the S-ICD, requirement of analgesia by the patients after implantation was comparable in the two groups.

Over the long-term, Kobe and collaborators analyzed the impact of the S-ICD position on patients' quality of life. Sixty S-ICD and 60 case-controlled TV-ICD patients were compared in terms of quality of life and post-traumatic stress disorders. This study revealed equal or even better physical well-being of patients with the S-ICD. Posttraumatic stress disorders occurred in almost $15 \%$ of ICD patients irrespective of the 
type of system [17]. In fact, the S-ICD does not limit arm movements which makes the device especially suitable for younger and active individuals.

Similarly to our study, a comparable mean length of stay after S-ICD implantation with both ICD systems has been described by other authors $[16,17]$. However, in our study, careful analysis of the reasons for prolonged hospitalization following a device implant, confirms that need of prolonged pain management was not among the reasons for prolonged hospitalization in any of the treatment arms.

\subsection{Limitations}

This study presents with some limitations which should be highlighted. First, this was a single-center non-randomized experience, with a small sample size. Second, the study was only exploratory in nature and was only powered to show major differences in the comparisons (e.g. a 2-fold or higher increase in any of the assessed outcomes). Lastly, the use of general anesthesia for TV-ICDs implantation is a specific policy of our center which cannot be generalized, and could have influenced the post-operative pain in this group of patients. In many centers, TV-ICDs implantations are mainly performed under deep sedation, which was not the case here. The availability of an anesthetist may limit the use of the S-ICD which is mostly implanted under general anesthesia. However, we believe that using general anesthesia for both TV-ICDs and S-ICDs made the comparison more relevant for the purpose of this study.

\section{Conclusions}

We observed a comparable performance of the S-ICD when compared to the TV-ICD with regard to efficacy and safety. Furthermore, our data on prescribed analgesia on- 
demand suggest that recovery period following the S-ICD implant is not more painful than with TV-ICDs, and that duration of hospitalization is also comparable.

Disclosures: S. Boveda is consultant for Medtronic, Boston Scientific, and Livanova. JP. Albenque is consultant for Abbott and Biosense Webster.

Fundings : none 


\section{References}

1. Bardy GH, Smith WM, Hood MA, Crozier IG, Melton IC, Jordaens L, et al. An entirely subcutaneous implantable cardioverter-defibrillator. N Engl J Med. 2010;363:36-44.

2. Cappato R, Ali H. Sudden cardiac death: New approaches for implantable cardioverter-defibrillators (ICDs). Int J Cardiol. 2017 Jun 15;237:38-41.

3. Lambiase PD, Barr C, Theuns DA, Knops R, Neuzil P, Johansen JB, et al. EFFORTLESS Investigators. Worldwide experience with a totally subcutaneous implantable defibrillator: early results from the EFFORTLESS S-ICD Registry. Eur Heart J. 2014 Jul 1;35(25):1657-65.

4. Priori SG, Blomström-Lundqvist C, Mazzanti A, Blom N, Borggrefe M, Camm J, et al. 2015 ESC Guidelines for the management of patients with ventricular arrhythmias and the prevention of sudden cardiac death: The Task Force for the Management of Patients with Ventricular Arrhythmias and the Prevention of Sudden Cardiac Death of the European Society of Cardiology (ESC). Endorsed by: Association for European Paediatric and Congenital Cardiology (AEPC). Eur Heart J 2015; 36:2793-867.

5. Boveda S, Lenarczyk R, Haugaa K, Fumagalli S, Madrid AH, Defaye P, et al. Implantation of subcutaneous implantable cardioverter defibrillatorsin Europe: results of the European Heart Rhythm Association survey. Europace 2016;18(9):1434-9.

6. Yancy CW, Jessup M, Bozkurt B, Butler J, Casey DE Jr, Colvin MM, et al. 2017 ACC/AHA/HFSA Focused Update of the 2013 ACCF/AHA Guideline for the Management of Heart Failure: Report of the American College of Cardiology/American Heart Association Task Force on Clinical Practice Guidelines 
and the Heart Failure Society of America. J Am Coll Cardiol. 2017 Apr 21. pii: S0735-1097(17)37087-0. doi: 10.1016/j.jacc.2017.04.025. [Epub ahead of print]

7. Healey JS, Hohnloser SH, Glikson M, Neuzner J, Mabo P, Vinolas X, et al. ; Shockless IMPLant Evaluation [SIMPLE] investigators. Cardioverter defibrillator implantation without induction of ventricular fibrillation: a single-blind, noninferiority, randomised controlled trial (SIMPLE). Lancet. 2015 Feb 28;385(9970):785-91.

8. Buchanan GL, Chieffo A, Mehilli J, Mikhail GW, Mauri F, Presbitero P, et al. Women In Innovation Group. The occupational effects of interventional cardiology: results from the WIN for Safety survey. EuroIntervention. 2012;8:658-63.

9. Jacob S, Boveda S, Bar O, Brézin A, Maccia C, Laurier D, et al. Interventional cardiologists and risk of radiation-induced cataract: results of a French multicenter observational study. Int J Cardiol. 2013;167:1843-7.

10. Moss AJ, Schuger C, Beck CA, Brown MW, Cannom DS, Daubert JP, et al. Reduction in inappropriate therapy and mortality through ICD programming. $\mathrm{N}$ Engl J Med, 2012; 367: 2275-2283.

11. Burke MC, Gold MR, Knight BP, Barr CS, Theuns DA, Boersma LV, et al. Safety and efficacy of the totally subcutaneous implantable defibrillator: 2-year results from a pooled analysis of the IDE study and EFFORTLESS registry. J Am Coll Cardiol 2015; 65:1605-15.

12. Mesquita J, Cavaco D, Ferreira A, Lopes N, Santos PG, Carvalho MS, Haas A, Costa F, Carmo P, Morgado F, Adragão P, Mendes M. Effectiveness of subcutaneous implantable cardioverter-defibrillators and determinants of inappropriate shock delivery. Int J Cardiol. 2017 Apr 1;232:176-180. 
13. Gold MR, Theuns DA, Knight BP, Sturdivant JL, Sanghera R, Ellenbogen KA, et al. Head-to-head comparison of arrhythmia discrimination performance of subcutaneous and transvenous ICD arrhythmia detection algorithms: the START study. J Cardiovasc Electrophysiol. 2012 Apr;23(4):359-66.

14. Honarbakhsh S, Providencia R, Srinivasan N, Ahsan S, Lowe M, Rowland E, et al. A propensity matched case-control study comparing efficacy, safety and costs of the subcutaneous vs. transvenous implantable cardioverter defibrillator. Int J Cardiol. 2017 Feb 1;228:280-285.

15. Brouwer TF, Yilmaz D, Lindeboom R, Buiten MS, Olde Nordkamp LR, Schalij MJ, et al. Long-Term Clinical Outcomes of Subcutaneous Versus Transvenous Implantable Defibrillator Therapy. J Am Coll Cardiol. 2016 Nov 8;68(19):20472055.

16. Köbe J, Reinke F, Meyer C, Shin DI, Martens E, Kääb S, et al. Implantation and follow-up of totally subcutaneous versus conventional implantable cardioverterdefibrillators: a multicenter case-control study. Heart Rhythm. 2013 Jan;10(1):29-36.

17. Köbe J, Hucklenbroich K, Geisendörfer N, Bettin M, Frommeyer G, Reinke F, et al. Posttraumatic stress and quality of life with the totally subcutaneous compared to conventional cardioverter-defibrillator systems. Clin Res Cardiol(2016): 1-5. 